\title{
Design and Implementation of Marketing System Based on Cluster of Target Customers
}

\author{
Xiaowei Li \\ \{lixiaoweiwh@yeah.net\} \\ Faculty of business, Wuhan Polytechnic, Wuhan 430074,China
}

\begin{abstract}
The paper gives a detail solution to build the telecommunication marketing system: first imported from various telecom production system of business data, built of aggregated data center of each system and the data were necessary regular; then, marketing for specific tasks, based on the characteristics of customer consumption analysis, find out the consumption characteristics of the target customers, and navigate to the target customers of marketing activities, and gives the corresponding marketing suggestions, and provide management and report module to support decision analysis. At last, the process of software design and development of marketing system is described in detail. In order to solve the marketing problems faced by telecom marketing, the marketing system is developed. In this paper, the design and implementation of telecom marketing system as the core, in order to focus on the positioning and recommendation of the target customers in the active marketing of telecom. The system in the use of data mining technology based, from massive customer data mining user groups, combined with the existing packages and tariff, and strive to accurately find the marketing opportunity to target customers, and to the user recommend a variety of packages or and meet the needs of users and to allow users to benefit from, the system will automatically generate marketing scripts to support the marketing executives who carry out the sale.
\end{abstract}

Keywords: Telecom marketing; data mining; target customer clustering; decision support

\section{Introduction}

With the rapid development of information technology and network technology, people are going to enter the era of cloud computing. At the same time, the popularization of computer and communication has brought a new business channel to the existing economic market, and has gradually formed a new business model. Enterprises can be very convenient with in the network, for a series of business activities by using electronic information means, to strengthen the communication and contact between the enterprise and the customer, and through active marketing in the existing enterprise internal marketing network, build marketing recommendation system in order to achieve anytime, anywhere. Under this kind of marketing mode, enterprises can not only active quickly collect customers of all kinds of information and customers to understand the real the most urgent needs[1], but also to active marketing behavior to influence the customer's consumption behavior and choose to buy, to help enterprises to open up emerging markets. On the one hand, to achieve a business to sell products as the core to meet customer needs as the core functions of the transformation, on the 
other hand is also convenient for customers to choose the most suitable for their products. Actually, the major operators in the construction of electronic channels, continuous innovation, basic hardware and technical construction progress quickly, but, in the service of electronic channels, marketing consciousness has lagged behind, thus forming electronic information channels are sluggish situation. Years committed to the construction of electronic channel operators in Shenzhen Ying source technology that: This is the only way to mature ecommerce. At present, the proportion of foreign advanced operators of electronic channels carrying the business has more than $90 \%$, a lot of operating costs. Their experience is worth our using for reference -- except in the infrastructure and technology innovation through of user information collection, behavior research, the effective improvement and integration of electronic channels is also very important. The construction of electronic channels is two-way and interactive, both by means of marketing to promote innovative electronic service channels and change the user the traditional use of the habit, but also the use of the user feedback to improve their services, business to break through the bottleneck, obtained the rapid development.

\section{Related Theories and Techniques}

According to the market need to organize the production of products, and by means of marketing to the needs of the customer is called marketing. In countries with different political, economic, and cultural, marketing should not be the same. Even in the same country, in the consumer goods industry, B2B industry (to business industries business) and service industry, marketing is also different. In the same industry, different companies have different marketing methods. Marketing is the subject of how companies find, create and deliver value to meet the needs of a certain target market, and to gain profit. Marketing is used to identify the need to identify, define, measure the size of the target market and profit potential, to find the most suitable for enterprises to enter the market segments and suitable for the market segments of the market supply [2].

\subsection{Market Segmentation Theory}

A good marketing strategy must be able to meet the actual market. Market analysis is the basis of product design and marketing strategy design. Market segmentation is the base of product differentiation design and differentiated marketing strategy. Therefore, subdivision theory is the most basic theoretical basis for the optimization of telecom marketing strategy.

Market segmentation is also called market segmentation (segmentation market), is the United States marketing professor Smith Wendell first put forward. Market segmentation theory is based on a the most common view, namely all consumers and is not the same, a company in marketing or make different marketing plans for different groups of consumer objects, or is just launched a marketing campaign to refer to a certain group. Market segmentation admitted in the multiple choice in the context of the market, consumers due to the differences between the various factors, also appears diversified, any single strategy corresponding to all the different consumers planning activities, is not a good strategic choice, and market segmentation in nature for rapidly improve the organization and management level of a large, diversified provides a practical possibility. The more mature products to market segmentation, based on market segmentation to find the corresponding customer market, for 
the customer market segments to take different marketing strategies. Only in this way can we find the customer base that is needed to bring the revenue growth point to the enterprise in the saturation or new market and the competitive market.

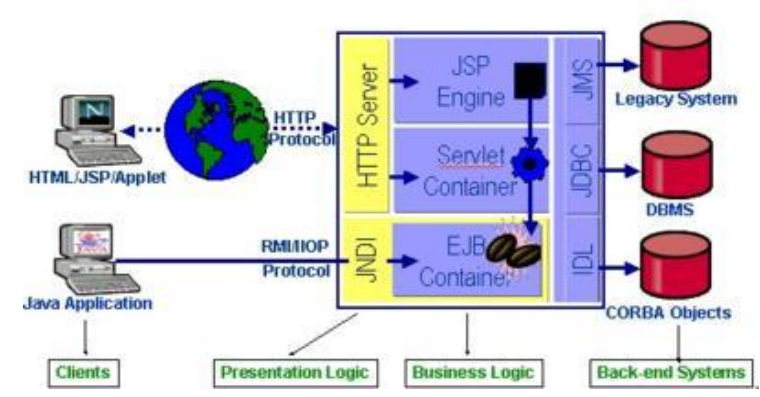

Fig. 1. System overall topology

\subsection{Clustering Analysis Algorithm}

Cluster analysis refers to the physical or abstract object set is divided into a number of classes of analysis process, in which the class of objects within each other acquaintance, class and class are unfamiliar with each other. The goal of cluster analysis is to collect and collate data on the basis of similarity. Clustering is an important human behavior, the so-called "Like attracts like., Birds of a feather flock together". Cluster analysis is derived from many disciplines, including mathematics, computer science, statistics, biology and economics, etc.. In different application areas, many clustering techniques have been developed. These techniques are used to describe the data, to measure the similarity between different data, and to classify the data into different clusters. Clustering enhances people's understanding of the objective world, and it is a prerequisite for the description of the concept and the analysis of the deviation. Clustering analysis data objects do not take into account the known class label [3]. In general, the training data do not provide a class label, because do not know where to start. Clustering can be used to produce this kind of tags. Object based on the principle of "maximizing the similarity within the class, minimizing the similarity between classes", the principle of clustering or grouping. That is, the clustering of objects so that the object in a class has a high similarity, which is not similar to the objects in other classes. Each cluster that is formed can be viewed as an object class, which can be derived from the rule. Clustering is also easy to formation taxonomy (classification system), the observed content is organized into a hierarchical structure, the organization of similar events together.

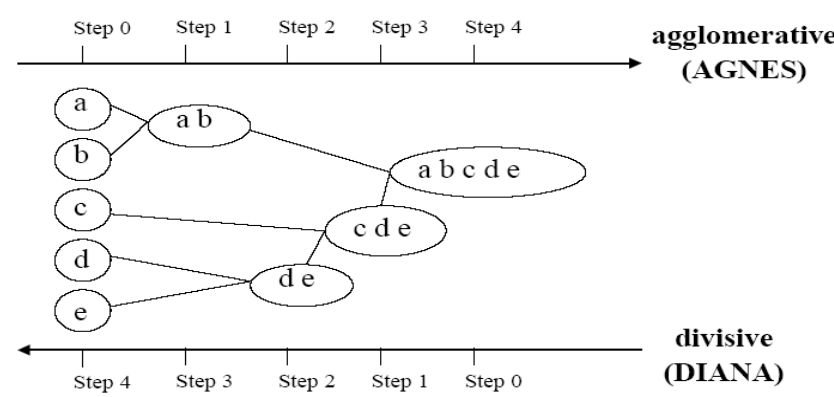


Fig. 2. Sketch of structure of clustering algorithm

\subsection{Data Mining Technology}

Data mining (DM), is from the stored in the database, data warehouse, or other information repositories of large amounts of data to obtain valid, novel, potentially useful, and ultimately understandable patterns of non trivial process. Simply speaking, data mining refers to extracting or "mining" knowledge from a large amount of data. In recent years, data mining caused the great attention of the information industry, the commercial areas, and society as a whole, the main reason is along with the computer technology, especially the development of storage technology, data is generated and stored more and more accumulated huge amounts of data, and is in urgent need of these data into useful information and knowledge, is widely used in various industries, including market analysis, fraud detection, customer retention, product control and scientific exploration and so on. Today, the database technology has developed mature and has been widely recognized and applied, online transaction processing (OLTP) is an effective method to query as read-only transactions, on the relationship between technology development and the relationship between technology as the effective storage of large amounts of data, the retrieval and management of main tools are widely recognized has made important contributions. Advances in computer hardware technology steadily, surprising, resulting in the supply of a large number of powerful and the price is suitable for the computer, data collection device and storage medium, promotes the emergence of data warehouse technology. Data warehouse is a kind of multi - heterogeneous data source in a single site to a unified model of the organization of the repository [4], in order to support management decisions. On the basis of the on-line analytical processing (OLAP) is a kind of analysis technology, which has the ability of collecting, merging and gathering functions, and viewing information from different dimensions. However, a new problem has emerged, that is, data rich, information poor. Massive data is stored in data warehouse, there is no powerful tool, it is very difficult to understand them, has exceeded the ability of people. Under the background of such business requirement, the data mining technology has emerged. Data mining tools for data analysis, you can find an important data model, the business strategy, knowledge base, science and some research to make a great contribution. "Diapers and beer" story is undoubtedly the best embodiment of the value of data mining. According to conventional thinking, diapers and beer is not the wind horse cow and, if not the use of data mining technology for a large number of transaction data mining analysis, WAL-MART is not possible to find the intrinsic value of the data of the law.

\section{Target Customer Clustering}

Through a section of the user data integration, we can of data is performed to establish the clustering model, $\mathrm{K}$ - means algorithm the dynamic division of thought, given a $\mathrm{n}$ a tuple or recorded data set, splitting method to construct K packet, every packet on behalf of a cluster, $\mathrm{K}<\mathrm{N}$ and the $\mathrm{K}$ packet to meet two conditions, namely each packet contains at least one data record and each data record belongs to and belong to a group. Need to determine some parameters to determine the $\mathrm{K}$ value, that is, the number of clusters, while the need to determine the $\mathrm{K}$ initial cluster center. Different initial cluster centers will lead to different clustering results, through a certain degree of the abnormal value, but there is inevitably the 
outlier removal is not thorough, so if the randomly selected initial clustering center method still has chosen to outliers risks, to outliers as the initial cluster centers will have the total number of than a few small class, affect the clustering performance. So the distance between the better initial cluster centers should be large enough. There are two main methods to select the initial cluster centers: one is the synthetic cluster center, the two is to choose the special actual observation point as the cluster center. Literature with eight different selection of the initial cluster centers were studied, respectively synthesized clustering center and the choice of the actual clustering center to do the experiment, and compared the effect of a variety of options. In this paper, random method is used to select $\mathrm{K}$ aggregation centers, and the random method is the most commonly used method in practice, because it is a random selection of $\mathrm{K}$ observations as the initial aggregation point combination, the results are often local optimal. Therefore, each $\mathrm{k}$ can choose different initial aggregation points, run several times, and take multiple runs of the Ek minimum clustering results for the final results. Run several times in this study [5].

\section{Conclusion}

In this paper, the telecommunications marketing system as the background, a detailed description of the system design and implementation process, and combined with data mining algorithm to guide marketing, to achieve a certain effect. The design process of the system is based on the actual needs of the telecommunications, can be divided into three modules: marketing module, management module, as well as the report module. System is mainly to solve the telecommunications marketing personnel to some problems in the actual operation, such as the need to go to the basic information of a user query in multiple heterogeneous systems, information consumption, information packages, this system integrated system of the underlying data, and according to the custom of marketing to set the interface show, but also related to the other related number with a user, this association in the actual process of marketing plays a very good effect. And to solve a marketing problem is how to find customers in the telecommunications users. The system through the demand analysis, the design of the marketing module, management module and report module, and through the data model design, provides an extension of dynamic user access and dynamic combination of recommended packages, and other functions. System can enhance the ability of data statistics and analysis, marketing activities of target customers positioning have more scientific method, strengthen the management personnel to monitor the process of marketing, through a unified data integration process, reducing the repeated work in the process of data in the past, and improve the efficiency of data access, unified management of marketing activities, strengthening the coordination of various marketing activities, effectively avoid the customers a disturbing phenomenon. In view of the customer group's method, this article uses the clustering algorithm to carry on the classification to the user, and the classification result is introduced into the marketing user database, the distribution to the marketing personnel. Before the implementation of the marketing system, telecommunication marketing work is difficult to carry out, marketing target data accuracy rate is very low, marketing success rate of only $2 \%-10 \%$; after the implementation of the marketing system. The success rate of marketing up to $15 \%-30 \%$, achieved significant results. 


\section{References}

[1] Wen Yanping, the history of kaiwah. Network marketing performance evaluation index system of. information magazine. No. 11. (2014)

[2] Geng Xiaoyuan, Zhang, Yan Yi. Improved k-means algorithm in telecom customer segmentation application. Computer technology and development. No.05. (2012)

[3] Lian Fengna, Wu Jinlin, Tang Qi. An improved K-means clustering algorithm. computer and information technology. No. 01. (2015)

[4] Liang Fang. 96688 target customers positioning to help companies find the most valuable customers in Guangdong. communication technology . No. 10, (2014)

[5] Jiang Ronghua, Deng Minhui. E-mail marketing target customer optimization research. public technology. No. 02. (2013) 